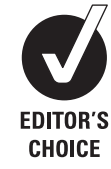

CHOICE

\title{
Statin use is associated with reduced incidence and progression of knee osteoarthritis in the Rotterdam study
}

\author{
S Clockaerts, ${ }^{1-3}$ G J V M Van Osch,,${ }^{1,4}$ Y M Bastiaansen-Jenniskens, ${ }^{1}$ J A N Verhaar, ${ }^{1}$ \\ F Van Glabbeek, ${ }^{2}$ J B Van Meurs, ${ }^{5}$ H J M Kerkhof, ${ }^{5,6}$ A Hofman, ${ }^{3,6}$ B H Ch Stricker, 3,5,7,8 \\ S M Bierma-Zeinstra ${ }^{1,9}$
}

\begin{abstract}
'Department of Orthopaedics, Erasmus MC, University Medical Center, Rotterdam, The Netherlands ${ }^{2}$ Department of Orthopaedic Surgery and Traumatology, University Hospital of Antwerp, Edegem, Belgium ${ }^{3}$ Department of Epidemiology, Erasmus MC, University Medical Center, Rotterdam, The Netherlands ${ }^{4}$ Department of Otorhinolaryngology, Erasmus MC, University Medical Center, Rotterdam, The Netherlands

${ }^{5}$ Department of Internal Medicine, Erasmus MC University Medical Center, Rotterdam, The Netherlands ${ }^{6}$ Netherlands Genomics Initiative (NGI)-Sponsored Netherlands Consortium for Healthy Aging (NCHA), The Netherlands

${ }^{7}$ Department of Medical Informatics, Erasmus MC, University Medical Center, Rotterdam, The Netherlands ${ }^{8}$ Inspectorate of Healthcare, The Hague, The Netherlands ${ }^{9}$ Department of General Practice, Erasmus MC, University Medical Center, Rotterdam, The Netherlands
\end{abstract}

\section{Correspondence to} Dr B H Ch Stricker, Department of Epidemiology, Erasmus MC, University Medical Center,

P0 Box 1738,

Rotterdam $3000 \mathrm{DR}$

The Netherlands;

b.stricker@erasmusmc.nl

Accepted 10 June 2011 Published Online First 11 October 2011

\begin{abstract}
Background Osteoarthritis is the most frequent chronic joint disease causing pain and disability. Besides biomechanical mechanisms, the pathogenesis of osteoarthritis may involve inflammation, vascular alterations and dysregulation of lipid metabolism. As statins are able to modulate many of these processes, this study examines whether statin use is associated with a decreased incidence and/or progression of osteoarthritis.
\end{abstract}

Methods Participants in a prospective populationbased cohort study aged 55 years and older $(n=2921)$ were included. $x$-Rays of the knee/hip were obtained at baseline and after on average 6.5 years, and scored using the Kellgren and Lawrence score for osteoarthritis. Any increase in score was defined as overall progression (incidence and progression). Data on covariables were collected at baseline. Information on statin use during follow-up was obtained from computerised pharmacy databases. The overall progression of osteoarthritis was compared between users and non-users of statins. Using a multivariate logistic regression model with generalised estimating equation, $\mathrm{OR}$ and $95 \% \mathrm{Cl}$ were calculated after adjusting for confounding variables.

Results Overall progression of knee and hip osteoarthritis occurred in $6.9 \%$ and $4.7 \%$ of cases, respectively. The adjusted OR for overall progression of knee osteoarthritis in statin users was $0.43(95 \% \mathrm{Cl} 0.25$ to $0.77, p=0.01$ ). The use of statins was not associated with overall progression of hip osteoarthritis.

Conclusions Statin use is associated with more than a $50 \%$ reduction in overall progression of osteoarthritis of the knee, but not of the hip.

Osteoarthritis is the most common form of arthropathy. It affects $9.6 \%$ of men and $18 \%$ of women aged 60 years or older and is the leading cause of disability in older people. ${ }^{2}$ The aetiology of osteoarthritis is not completely understood. Besides genetic variation and biomechanical mechanisms, inflammation can lead to cartilage matrix breakdown, synovial hypertrophy, subchondral bone sclerosis and osteophyte formation. ${ }^{3-5}$ The pathogenesis of osteoarthritis might also involve altered lipid metabolism and vascular pathology. ${ }^{6-8}$

Current treatment of osteoarthritis consists of exercise therapy and lifestyle adjustment, with pharmacotherapeutic treatment of symptoms when needed. However, the therapeutic efficacy of this treatment is small to moderate. ${ }^{9}$ Until now, there is no disease-modifying compound for osteoarthritis. ${ }^{59}$ In the past few decades, drug research and development has mainly focused on articular cartilage, even though the whole joint is affected in osteoarthritis and the disease process may also be influenced by systemic factors.

In addition to lowering the circulating level of low-density lipoproteins, statins have a broad range of biological effects including anti-inflammatory properties in different cell types. In-vitro studies revealed that statins have antioxidative effects, decrease the production of matrix metalloproteinases, interleukins and increase the production of aggrecan and collagen type II in chondrocytes. ${ }^{10}$ In synovial cells, statins also decrease the production of matrix metalloproteinases, interleukins, chemokines and induce apoptosis in synovial fibroblasts. ${ }^{10}$ Furthermore, statins might influence osteoarthritis by their ability to inhibit osteoclastogenesis, to stimulate bone formation and to counteract possible underlying mechanisms of osteoarthritis, such as decreasing plasma low-density lipoprotein levels, vascular pathology and systemic inflammation. ${ }^{11-13}$

Statins are now frequently used as lipid-lowering drugs. As statins seem capable of targeting different underlying mechanisms of osteoarthritis, these drugs have been suggested as possible diseasemodifying drugs for osteoarthritis. ${ }^{614-16}$ However, reliable data to support this assumption are lacking. Therefore, this study examines the association between statin use and the incidence and/or progression of knee and/or hip osteoarthritis in a large population-based study.

\section{METHODS \\ Study population}

The Rotterdam study is a prospective populationbased cohort study set up to investigate the occurrence and determinants of diseases in an ageing population. ${ }^{17}$ All 10275 inhabitants aged 55 years and older who have lived for at least 1 year in the Ommoord district of the city of Rotterdam were asked to participate. The response rate was $78 \%$, which means that 7983 individuals responded. The Medical Ethics Committee of the Erasmus MC University Medical Center approved the study and 
all participants gave written informed consent. Baseline measurements were obtained from 1990 to 1993 and consisted of a home interview and visits to the research centre for physical examinations. Follow-up data were collected during a follow-up visit from 1996 to 1999 . The present study includes participants for whom radiographs of knees and hips at baseline and follow-up were present and scored. Patients with Bechterew's disease, rheumatoid arthritis, gout, upper leg fractures (for knee osteoarthritis) and hip fractures (for hip osteoarthritis) were excluded.

\section{Exposure assessment}

Within the Ommoord district, seven fully computerised pharmacies are linked to one computer network. Ninety-eight per cent of the participants filled their prescriptions in one of these pharmacies during the period from baseline to follow-up. All data on dispensed drugs are available in computerised form on a day-today basis. Information is available on the date of prescribing, the total amount of drug units on each prescription, the prescribed daily number of units, the product name of the drugs and the anatomical therapeutic chemical code. ${ }^{18}$ For each participant, the use of statins was extracted for the period between baseline and the follow-up visit. To avoid non-differential misclassification of exposure, we excluded participants who were taking statins at baseline, as no data were available on the duration of the use of statins during the period before baseline. Subjects with a cumulative use of less than 120 days and/or a daily intake of less than $50 \%$ of the recommended daily adult dose for the treatment of hypercholesterolemia in The Netherlands were considered as non-users because below this period and dose a substantial protective effect on a slowly progressive disease such as osteoarthritis can not be expected. To investigate the effects of increasing cumulative exposure, we defined a priori three intervals of statin use for subjects with an average daily intake of $50 \%$ or greater: 1-119 days, 120-364 days and 365 days or greater. Lipophilic statins (in this study atorvastatin, simvastatin, fluvastatin and lovastatin) and hydrophilic statins (pravastatin) have a common mechanism of action, but differ in terms of absorption, distribution, metabolism and excretion. ${ }^{19}$ Therefore, we distinguished between lipophilic and hydrophilic statins in the additional analyses.

\section{Outcome assessment}

Weight-bearing anteroposterior radiographs of the knee and hip were obtained at $70 \mathrm{kV}$, a focus of 1.8 , and a focus to film distance of $120 \mathrm{~cm}$, applying a Fuji high resolution G $35 \times 43 \mathrm{~cm}$ film. The knee was extended with the patella in a central position. Radiographs of the pelvis were obtained with both feet in $10^{\circ}$ internal rotation and the x-ray beam centred on the umbilicus. Radiographs were assessed following the Kellgren and Lawrence (K\&L) grading system, ${ }^{20-23}$ atlas based, in five (0-4) grades (table 1). In addition, we defined a joint prosthesis as grade $5 .^{24}$ Each radiograph was scored by one trained reader of in total seven readers. The interrater reliability between two of the seven readers was tested in a random set of $10 \%$ of radiographs. The $\kappa$ value (cut-off value: K\&L score $\geq 2$ ) was $0.71(95 \%$ CI 0.66 to 0.76 ) for the knee and 0.74 (95\% CI 0.70 to 0.78 ) for the hip. The radiographs at baseline and follow-up were read without knowledge of the clinical status of the participants or without knowledge of the research hypothesis or exposure status of the participants. Left and right radiographs were grouped per subject and read by pairs in chronological order. ${ }^{25}$ As there is no consensus on the definition of incidence and progression, we combined both in one definition for the overall progression of osteoarthritis. This was defined as an increase in the K\&L score between baseline and follow-up of 1 or more. In the case of a baseline score 0 , overall progression was defined as an increase of 2 or more. Patients with scores 4 or 5 at baseline were left out of the analysis. For transparency we also reported on previously used outcomes for incidence and progression separately (incidence defined as score 0 or 1 at baseline and a follow-up score of 2 or more; progression defined as a baseline score of 1, 2, 3 and an increase of 1 or more). ${ }^{26}$

\section{Co-factors}

Trained interviewers gathered information on medical history and risk factors for chronic diseases. Participants were invited to visit the research centre for clinical examinations and laboratory assessments. The following information was collected and defined as possible confounders for the present study: gender, age, body mass index (BMI; weight $(\mathrm{kg}) /$ height $\left(\mathrm{m}^{2}\right)$ ), femoral bone mineral density (BMD; measured by dual-energy x-ray absorptiometry; Lunar DPX-L densitometer, Lunar Corporation, Madison, WI), total cholesterol/high-density lipoprotein (HDL) ratio (serum total cholesterol determined by an enzymatic procedure, HDL measured after precipitation of non-HDL cholesterol), current smoking (self-reported), diabetes mellitus (use of glucose-lowering medication or non-fasting random or postload glucose levels exceeding $11.0 \mathrm{mmol} / \mathrm{l})$, peripheral artery disease

Table 1 Kellgren and Lawrence scores for osteoarthritis of the knee and the hip.

\begin{tabular}{|c|c|c|}
\hline Grade & Knee & Hip \\
\hline \multicolumn{3}{|l|}{$\overline{0}$} \\
\hline No osteoarthritis & No features of osteoarthritis & No features of osteoarthritis \\
\hline \multicolumn{3}{|l|}{1} \\
\hline Doubtful & $\begin{array}{l}\text { Doubtful narrowing of joint space and possible } \\
\text { osteophytic lipping }\end{array}$ & $\begin{array}{l}\text { Possible narrowing of joint space medially and } \\
\text { possible osteophytes around the femoral head; or } \\
\text { osteophytes alone }\end{array}$ \\
\hline \multicolumn{3}{|l|}{2} \\
\hline Mild & $\begin{array}{l}\text { Definite osteophytes and possible narrowing of } \\
\text { joint space }\end{array}$ & $\begin{array}{l}\text { Definite narrowing of joint space inferiorly, definite } \\
\text { osteophytes and slight sclerosis }\end{array}$ \\
\hline \multicolumn{3}{|l|}{3} \\
\hline Moderate & $\begin{array}{l}\text { Moderate multiple osteophytes, definite } \\
\text { narrowing of joint space and some sclerosis and } \\
\text { possible deformity of bone ends }\end{array}$ & $\begin{array}{l}\text { Marked narrowing of joint space, definite } \\
\text { osteophytes, some sclerosis and cyst formation, and } \\
\text { deformity of the femoral head and acetabulum }\end{array}$ \\
\hline \multicolumn{3}{|l|}{4} \\
\hline Severe & $\begin{array}{l}\text { Large osteophytes, marked narrowing of joint } \\
\text { space, severe sclerosis and definite deformity of } \\
\text { bone ends }\end{array}$ & $\begin{array}{l}\text { Gross loss of joint space with sclerosis and cysts, } \\
\text { marked deformity of femoral head and acetabulum } \\
\text { and large osteophytes }\end{array}$ \\
\hline
\end{tabular}


(ankle/brachial index $<0.9$ in at least one leg), arterial hypertension (systolic blood pressure of $160 \mathrm{~mm} \mathrm{Hg}$ or higher, diastolic blood pressure of $95 \mathrm{~mm} \mathrm{Hg}$ or higher, or the use of antihypertensive drugs for hypertension), educational level and number of months between baseline and follow-up.

\section{Statistical analysis}

Baseline characteristics of statin users/non-users were compared using Student's t test and Pearson's $\chi^{2}$ test. When examining the association between statin use and osteoarthritis, a joint-based analysis of knees and hips was used with generalised estimating equations to fit the models for correlations between the right and left extremity in each individual. ${ }^{27}$ A multivariate logistic regression model including statin use and adjusting for confounding variables was fitted to calculate $\mathrm{OR}, 95 \% \mathrm{CI}$ and $\mathrm{p}$ values for the overall progression of knee and hip osteoarthritis. The definition of progression implies that participants are restricted (ie, conditioned) by the baseline presence of osteoarthritis. The estimates of an effect of exposure on outcome are therefore potentially biased. The effects of determinant on the estimate of outcome can only be assessed correctly by including all potential confounders in the multivariate analysis, thereby creating a model in which the conditioning by baseline is minimised. ${ }^{28}$ Therefore, we adjusted for all possible confounding variables based on the literature. We also adjusted for months between the baseline and follow-up visit, and for the baseline K\&L score. Subjects with missing values were excluded from the analysis. The dataset contained one $(0.03 \%)$ missing value for age and gender, 14 missing values (0.48\%) for BMI, 29 (0.99\%) for hypertension, five $(0.17 \%)$ for diabetes mellitus, $248(8.49 \%)$ for femoral BMD, 137 (4.69\%) for HDL/total cholesterol ratio, 18 (0.01\%) for education, $230(7.87 \%)$ for peripheral artery disease and 17 $(0.58 \%)$ for smoking. The category reflecting no use of statins was set as the reference. Results with a $\mathrm{p}$ value below 0.05 were considered statistically significant.

\section{RESULTS}

Of the total 7983 participants, 2921 were included in this study (figure 1). Osteoarthritis (K\&L score of $\geq 2$ ) was present in 677 knees $(12.5 \%)$ and 335 hips (5.8\%) at baseline, and in 939 knees $(17.5 \%)$ and 508 hips (8.8\%) at follow-up. After the exclusion of participants with fractures of the femur (for knee osteoarthritis) and the hip (for hip osteoarthritis), and with end-stage osteoarthritis or joint prosthesis, the overall progression of left or right

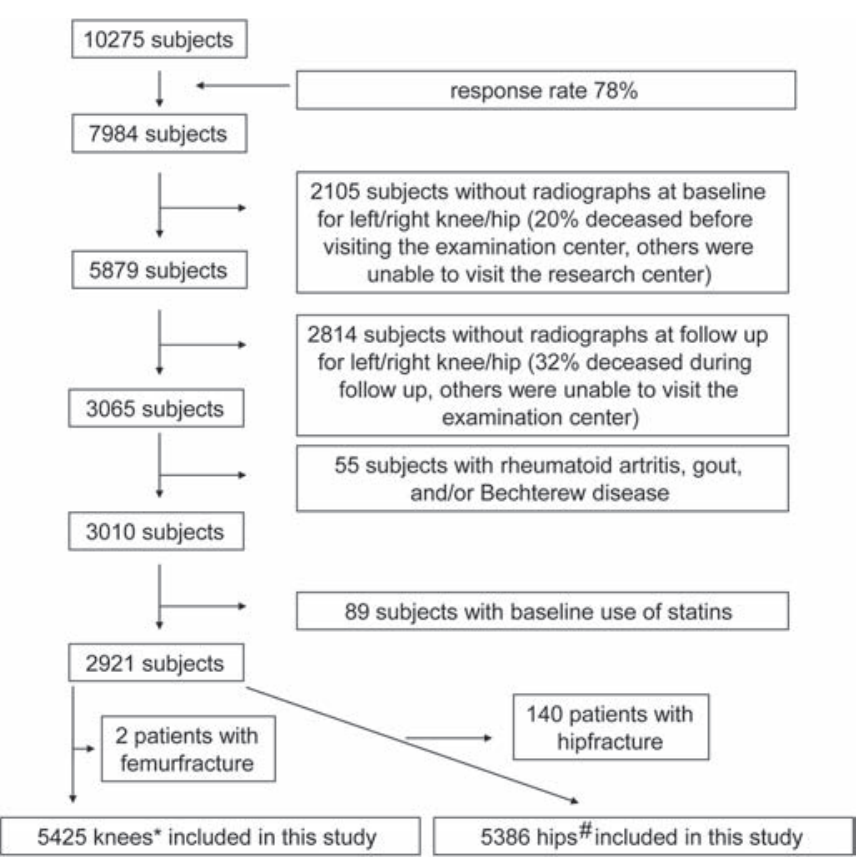

Figure 1 Flow chart showing subjects from eligibility to inclusion in the present study; 972 knees $\left(^{*}\right)$ and 919 hips $\left({ }^{\#}\right)$ were excluded from the multivariate analyses due to missing values in covariables.

Table 2 Characteristics of the study population $(n=2921)$

\begin{tabular}{|c|c|c|c|}
\hline \multirow[b]{2}{*}{ Variables } & \multirow{2}{*}{$\begin{array}{l}\text { Statin users }(n=317) \\
\text { Means (SD) or } n(\%)\end{array}$} & \multirow{2}{*}{$\begin{array}{l}\text { Non-users }(n=2604) \\
\text { Means (SD) or } n(\%)\end{array}$} & \multirow[b]{2}{*}{ p Value } \\
\hline & & & \\
\hline Age, years & $64.1(5.6)$ & $65.9(6.8)$ & $<0.001$ \\
\hline Female, \% & $178(56.3)$ & $1495(57.4)$ & 0.71 \\
\hline Body mass index, $\mathrm{kg} / \mathrm{m}^{2}$ & $26.4(3.48)$ & $26.27(3.4)$ & 0.45 \\
\hline Femoral bone mineral density, $\mathrm{g} / \mathrm{cm}^{2}$ & $0.9(0.13)$ & $0.85(0.13)$ & 0.11 \\
\hline Plasma total cholesterol/high-density lipoprotein ratio & $6.5(2.13)$ & $5.11(1.5)$ & $<0.001$ \\
\hline Arterial hypertension & $111(35.4)$ & $707(27.4)$ & 0.01 \\
\hline Peripheral artery disease & $30(10.1)$ & $230(9.6)$ & 0.80 \\
\hline Smoker & $79(25.2)$ & $539(20.8)$ & 0.07 \\
\hline Diabetes mellitus & $31(9.8)$ & $176(6.8)$ & 0.05 \\
\hline Higher education & $60(9.6)$ & $546(10.5)$ & 0.44 \\
\hline Follow-up period (months) & $77.7(4.3)$ & $77.78(4.5)$ & 0.70 \\
\hline Femur fracture & $0(0.0)$ & $2(0.1)$ & 0.62 \\
\hline Hip fracture & $10(3.2)$ & $130(5.0)$ & 0.15 \\
\hline \multicolumn{4}{|l|}{ Kellgren and Lawrence score $\geq 2$ of left knee } \\
\hline Baseline & $25(8.5)$ & $279(11.8)$ & 0.09 \\
\hline Follow-up & $34(11.8)$ & $383(16.4)$ & 0.04 \\
\hline \multicolumn{4}{|l|}{ Kellgren and Lawrence score $\geq 2$ of right knee } \\
\hline Baseline & $32(10.9)$ & $341(13.9)$ & 0.16 \\
\hline Follow-up & $38(13.1)$ & $481(19.7)$ & 0.01 \\
\hline \multicolumn{4}{|l|}{ Kellgren and Lawrence score $\geq 2$ of left hip } \\
\hline Baseline & $15(4.7)$ & $133(5.2)$ & 0.74 \\
\hline Follow-up & $20(6.3)$ & $198(7.7)$ & 0.37 \\
\hline \multicolumn{4}{|l|}{ Kellgren and Lawrence score $\geq 2$ of right hip } \\
\hline Baseline & $16(5.0)$ & $171(6.6)$ & 0.28 \\
\hline Follow-up & $26(8.2)$ & $264(10.3)$ & 0.24 \\
\hline
\end{tabular}


knee osteoarthritis in the study population was $6.9 \%$, and $4.7 \%$ for the hip joints.

Of this group, 317 (10.9\%) were defined as statin users. Statin users were younger, had higher plasma total cholesterol/HDL ratios, and more frequently reported arterial hypertension and diabetes mellitus than non-users (table 2). Between statin users and non-users there were no differences in gender, educational level, BMI, femoral BMD, those that reported smoking, and individuals with peripheral artery disease. The occurrence of hip fractures, femur fractures and the follow-up period was similar in both groups.

Statin use was defined as the use of statins for 120 days or greater, and $50 \%$ or greater of the daily recommended intake. Data are means (SD) or $\mathrm{n}(\%)$. Subjects with missing values were excluded from the analysis. Osteoarthritis is defined as a K\&L score of 2 or more. All subjects with baseline statin use, rheumatoid arthritis, gout, Bechterew disease, femur fracture (for knee osteoarthritis) and hip fracture (for hip osteoarthritis) were excluded.

The use of statins is associated with a reduction in the overall progression of knee osteoarthritis (OR 0.43 , 95\% CI 0.25 to $0.77, p=0.01$ ) after adjustment for the baseline K\&L score, possible confounders and months between baseline and follow-up (table 3).

Adjusted OR, 95\% CI and p values were computed by multivariate analysis with generalised estimating equations to account for left and right correlations. The OR were adjusted for the baseline K\&L score, gender, age, BMI, femoral BMD, total cholesterol/HDL ratio, current smoking, diabetes mellitus, peripheral artery disease, arterial hypertension, educational level and months between baseline and follow-up. Statin use was defined as the use of statins of 120 days or greater, and $50 \%$ or greater of the daily recommended intake. To analyse the effect of increasing cumulative exposure, three intervals of statin use were defined for subjects with an average daily intake of $50 \%$ or greater: 1-119, 120-364 and 365 days or greater, whereas all other subjects were defined as non-users.

These results were confirmed by analysing the effect of cumulative exposure to statin use (daily intake $\geq 50 \%$ ) in users of lipophilic statins (OR 0.50, 95\% CI 0.26 to 0.95 , $\mathrm{p}=0.03$ ). The data were underpowered to examine the association between hydrophilic statin use and the overall progression of knee osteoarthritis. To investigate whether the association between statin use and the overall progression of knee osteoarthritis is (or is not) due to confounding by indication, we computed models for the difference between statin users, minimal users (subjects that used statins $<120$ days or $<50 \%$ of the recommended daily adult dose) and non-users. There was a significant decrease in the overall progression of knee osteoarthritis in the user group (OR $0.44,95 \%$ CI 0.25 to $0.77, \mathrm{p}=0.01$ ) and no association in the minimal user group (OR 1.17, $95 \%$ CI 0.50 to $2.71, p=0.72$ ). Additional analyses with previously used definitions of incidence and progression ${ }^{26}$ also found significant associations between statin use and the incidence of osteoarthritis of the knee (OR $0.45,95 \%$ CI 0.24 to $0.85, \mathrm{p}=0.01$ ) and progression of osteoarthritis in the knee (OR 0.39, 95\% CI 0.20 to 0.75 , p=0.01).

In the hip joint, no associations were found between statin use and the overall progression of osteoarthritis (OR 1.10, 95\% CI 0.63 to $1.90, p=0.74)$. The incidence of hip osteoarthritis (OR $0.87,95 \%$ CI 0.46 to $1.67, \mathrm{p}=0.70$ ) or progression (OR $1.25,95 \%$ CI 0.70 to $2.21, p=0.45$ ) in the hip separately were also not associated with statin use (data not shown).

\section{DISCUSSION}

This population-based study demonstrates that the use of statins is associated with a decrease in the overall progression of osteoarthritis of the knee, but not of the hip.

For this study, we used a large population cohort study, the Rotterdam Study, in which all relevant clinical, radiological and pharmacological data were collected from a population at risk of the progression of osteoarthritis. Detailed pharmacological data for the total period were available in computerised databases of the local pharmacies. We included potential confounders that could influence the association between statin use and osteoarthritis, and adjusted for the baseline presence of osteoarthritis in our analyses of progression. ${ }^{28}$ Many of these covariables are characteristics of a cardiovascular and metabolic profile, because osteoarthritis is a disease with a multifactorial aetiology involving biomechanical, genetic, inflammatory, vascular and metabolic factors. 378

In this study, the overall progression of osteoarthritis was defined as the combination of the incidence and progression of existing osteoarthritis at baseline, because both outcome parameters can not be accurately defined based on radiographic examination alone. Although the Rotterdam Study is a large study, the number of statin users and subjects with overall progression were low. Using separate definitions of incidence and progression would have decreased the numbers even further. However, when we used those separate definitions of incidence and progression, we found similar results.

The use of statins was clearly associated with the reduced progression of knee osteoarthritis, whereas this was not the case for the hip joint. This difference can not be attributed to a lack of power, because the number of hips included was comparable to that of knee joints. The difference in effect on hip joints in the present study may indicate a difference in pathogenesis between the knee and the hip. As statins only target non-biomechanical mechanisms, our data may suggest that osteoarthritis of the knee is influenced more by metabolic factors such as the secretion of cytokines and adipokines by adipose tissue, vascular pathology in the subchondral bone or the direct effects of lipids on joint tissues ${ }^{6-8} 29$ than osteoarthritis of the hip. Together with changed mechanics especially in the knee

Table 3 Association between statin use and progression of knee and hip osteoarthritis

\begin{tabular}{|c|c|c|c|c|c|c|c|c|}
\hline & \multicolumn{4}{|l|}{ Knee osteoarthritis } & \multicolumn{4}{|l|}{ Hip osteoarthritis } \\
\hline & Progressors, N (\%) & Adjusted OR & $95 \% \mathrm{Cl}$ & p Value & Progressors, N (\%) & Adjusted OR & $95 \% \mathrm{Cl}$ & p Value \\
\hline Non-users & $288(7.3)$ & 1.00 & - & - & $177(4.5)$ & 1.00 & - & - \\
\hline Statin users & $15(3.0)$ & 0.43 & 0.25 to 0.77 & 0.01 & $21(4.0)$ & 1.10 & 0.63 to 1.90 & 0.74 \\
\hline \multicolumn{9}{|c|}{ Period of statin use (days) } \\
\hline Non-users & $346(7.2)$ & 1.00 & - & - & $228(4.8)$ & 1.00 & - & - \\
\hline $1-119$ & $53(6.3)$ & 1.77 & 0.71 to 4.44 & 0.22 & $3(4.1)$ & 1.05 & 0.20 to 5.62 & 0.95 \\
\hline $120-364$ & $3(2.4)$ & 0.30 & 0.08 to 1.19 & 0.09 & $6(4.8)$ & 1.05 & 0.38 to 2.88 & 0.92 \\
\hline$\geq 365$ & $18(4.1)$ & 0.49 & 0.26 to 0.92 & 0.03 & $17(3.6)$ & 1.00 & 0.51 to 1.98 & 0.92 \\
\hline
\end{tabular}


due to obesity, ${ }^{30}$ this might explain why BMI is a major risk factor for knee osteoarthritis, but not for the hip. ${ }^{31-33}$ Systemic factors such as disordered glucose metabolism, lipid metabolism and atherosclerosis have already been linked to knee osteoarthritis. Besides the potential role of cytokines and adipokines secreted by subcutaneous and visceral adipose tissue, there is also the presence of a large intra-articular fat pad that has been suggested to play an important role in knee osteoarthritis. ${ }^{12} 29$ It would have been worthwhile to investigate effect modification by obesity, age and gender by stratified analysis, but the data were underpowered to perform these extra analyses.

In the present study no association was found between statin use and hip osteoarthritis. An earlier study among 5678 woman (aged 65 years and older) on the association between statin use and radiographic hip osteoarthritis reported a higher incidence of hip osteoarthritis in the group of statin users over a period of 8 years; they also reported a trend towards a decrease in progression, albeit not significant. However, in that study, the multivariate models were not adjusted for confounding variables such as diabetes mellitus or the serum cholesterol level. Furthermore, statin use was based on statin prescriptions gathered by patients at follow-up and no data were available on statin intake during the follow-up period, or on the dose or period of intake. ${ }^{34}$ This is in contrast to our study, in which we obtained data on statin use from computerised databases of local pharmacies during the follow-up period. Another study investigated the association between statin use and osteoarthritis in a retrospective cohort study among the members of a 1.8 million health maintenance organisation in Israel, and found no significant association after long-term follow-up. In that study, however, they only investigated reported general osteoarthritis (based on the International Classification of Diseases, 9th revision, codes 715.x) without stratifying by joint, ${ }^{35}$ whereas we were able to investigate osteoarthritis of the knee and the hip separately, based on radiographic examination.

The use of anteroposterior radiographs of the knee in 20-30 flexion improves the reliability of joint space width compared with the straight leg radiographs used in the Rotterdam Study. ${ }^{36}$ Therefore, our reported association for the knee might be less precise than with the use of flexed knee radiographs. In addition, the radiographs were scored in known time order, which increases the sensitivity to change. As a result of the observational nature of the present study, selection bias, information bias or confounding are potential limitations. Although the high participation rate in the Rotterdam Study makes selection bias less likely, baseline hip and/or knee radiographs were available for approximately $74 \%$ of participants. In addition, because of a loss to follow-up, baseline and follow-up radiographs were available for only $38 \%$ of the 7984 initially included participants. Patients had to be mobile and motivated enough to come to the examination centre at baseline and follow-up, and had to survive the follow-up period to have radiographs at both time points. This caused a health-based selection bias. In the group with baseline radiographs compared to those without, subjects were younger (68 vs 78 years), there were fewer subjects with arterial hypertension $(33.8 \%$ vs $45.5 \%)$ and fewer people with diabetes mellitus $(9.8 \%$ vs $14.7 \%)$. There were also more men in the subjects with baseline radiographs $(41.7 \%$ vs $31.1 \%$ women). It is difficult to say how this has affected our findings, unless healthy participants show a better response to statins than unhealthy non-participants. Because of the prospective nature of this study, information bias is highly unlikely and, as we adjusted for all known confounders, our results are probably valid. Confounding by indication was investigated by calculating the risk estimates for those who used statins for less than 120 days. In this group, there was a slight but non-significant increase in the progression of knee osteoarthritis instead of a decrease. This suggests that confounding by indication would tend to underestimate a true protective effect. The statin users tended to have fewer K\&L scores of 2 or more at baseline. However, statin users were younger and there were fewer women in the statin users group. When performing statistical analysis with adjustment for these covariables, there was no longer a tendency towards lower K\&L scores at baseline in the statin user group (data not shown).

Statins may be a candidate for the pharmacological management of osteoarthritis as they are drugs capable of altering serum lipid levels, inflammatory pathways in endothelial cells, macrophages, chondrocytes, synoviocytes and they have protective effects on subchondral bone by inhibiting osteoclastogenesis and stimulating bone formation. ${ }^{10} 111437-41$ However, it should be noted that our study only indicates the disease-modifying effect of statins, and did not investigate differences in pain or disability between statin users and non-users. Additional studies on the use of statins as a potential therapy for osteoarthritis are required. It would be interesting to investigate the association between statin use and hand osteoarthritis, which is likely to be more metabolically influenced than knee osteoarthritis. ${ }^{42}$ The effect of statins on clinical outcome measures such as pain or disability should also be investigated.

In conclusion, we found that statin use is associated with a reduction in the overall progression of knee osteoarthritis after adjustment for potential confounding variables. Although these findings need to be replicated, they indicate that the causal association between statins and osteoarthritis should be investigated further.

Acknowledgements The authors are very grateful to the participants and staff from the Rotterdam Study, the participating general practitioners and the pharmacists. They would like to thank M Reijman, A Bergink, A van Vaalen, M Kool, JS Dekkers for the scoring of radiographs and $\mathrm{E}$ Oei for assistance.

Funding This study is financially supported by the Nuts-Ohra Foundation. SC received a scholarship from the University of Antwerp and the Anna Foundation. The Rotterdam Study is funded by the Erasmus Medical Center and Erasmus University in Rotterdam, The Netherlands Organization for Health Research and Development (ZonMw), the Research Institute for Diseases in the Elderly (RIDE), the Ministry of Education, Culture and Science, The Netherlands Genomics Initiative (NGI)/ Netherlands Consortium for Healthy Aging (NCHA) project no 050-060-810, the European Commission framework 7 programme TREAT-OA (grant 200800), the Ministry for Health, Welfare and Sports, the European Commission (DG XII) and the Municipality of Rotterdam.

\section{Competing interests None.}

Ethics approval This study was approved by the Medical Ethics Committee of the Erasmus MC University Medical Center.

\section{Patient consent Obtained.}

Contributors All authors mentioned in this study contributed to the conception and design, or analysis and interpretation of data, drafting of the article or revising it critically for important intellectual content and final approval of the version to be published.

Provenance and peer review Not commissioned; externally peer reviewed.

\section{REFERENCES}

1. Brooks PM. The burden of musculoskeletal disease - a global perspective. Clin Rheumatol 2006;25:778-81

2. Woolf AD, Pfleger B. Burden of major musculoskeletal conditions. Bull WHO 2003:81:646-56.

3. Goldring SR. Role of bone in osteoarthritis pathogenesis. Med Clin North Am 2009;93:25-35; xv.

4. Goldring SR, Goldring MB. The role of cytokines in cartilage matrix degeneration in osteoarthritis. Clin Orthop Relat Res 2004;427(Suppl):S27-36.

5. Hunter DJ, Felson DT. Osteoarthritis. BMJ 2006;332:639-42. 
6. Conaghan PG, Vanharanta H, Dieppe PA. Is progressive osteoarthritis an atheromatous vascular disease? Ann Rheum Dis 2005;64:1539-41.

7. Davies-Tuck ML, Hanna F, Davis SR, et al. Total cholesterol and triglycerides are associated with the development of new bone marrow lesions in asymptomatic middle-aged women - a prospective cohort study. Arthritis Res Ther 2009;11:R181.

8. Masuko K, Murata M, Suematsu N, et al. A metabolic aspect of osteoarthritis: lipid as a possible contributor to the pathogenesis of cartilage degradation. Clin Exp Rheumatol 2009;27:347-53.

9. Zhang W, Moskowitz RW, Nuki G, et al. OARSI recommendations for the management of hip and knee osteoarthritis. Part II: OARSI evidence-based, expert consensus guidelines. Osteoarthr Cartil 2008;16:137-62.

10. Lazzerini PE, Capecchi PL, Selvi E, et al. Statins and the joint: multiple targets for a global protection? Semin Arthritis Rheum 2011:40:430-46.

11. Bauer DC. HMG CoA reductase inhibitors and the skeleton: a comprehensive review. Osteoporos Int 2003;14:273-82.

12. Clockaerts S, Bierma-Zeinstra SM. Comment on: statins and the joint: multiple target for a global protection? Semin Arthritis Rheum 2011;40:588.

13. Nissen SE, Tuzcu EM, Schoenhagen P, et al. Statin therapy, LDL cholesterol, C-reactive protein, and coronary artery disease. N Engl J Med 2005;352:29-38.

14. Akasaki Y, Matsuda S, Nakayama K, et al. Mevastatin reduces cartilage degradation in rabbit experimental osteoarthritis through inhibition of synovial inflammation. Osteoarthr Cartil 2009;17:235-43.

15. Ghosh P, Cheras PA. Vascular mechanisms in osteoarthritis Best Pract Res Clin Rheumatol 2001;15:693-709.

16. Wu YS, Hu YY, Yang RF, et al. The matrix metalloproteinases as pharmacological target in osteoarthritis: statins may be of therapeutic benefit. Med Hypotheses 2007:69:557-9.

17. Hofman A, Breteler MM, van Duijn CM, et al. The Rotterdam Study: 2010 objectives and design update. Eur J Epidemiol 2009;24:553-72.

18. World Health Organization. Anatomical Therapeutic Classification (ATC) Index: including defined daily doses (DDD) for plain substances. Geneva: WHO, 1992

19. Schachter M. Chemical, pharmacokinetic and pharmacodynamic properties of statins: an update. Fundam Clin Pharmacol 2005;19:117-25.

20. Kellgren JH, Jeffrey MR, Ball J. The Epidemiology of Chronic Rheumatism. Atlas of Standard Radiographs of Arthritis. Oxford: Blackwell Scientific Publications 1963.

21. Kellgren JH, Lawrence JS. Radiological assessment of osteo-arthrosis. Ann Rheum Dis 1957;16:494-502

22. Reijman $\mathbf{M}$, Hazes JM, Pols HA, et al. Validity and reliability of three definitions of hip osteoarthritis: cross sectional and longitudinal approach. Ann Rheum Dis 2004;63:1427-33

23. Schiphof D, Boers M, Bierma-Zeinstra SM. Differences in descriptions of Kellgren and Lawrence grades of knee osteoarthritis. Ann Rheum Dis 2008;67:1034-6.

24. Spector TD, Hart DJ, Byrne J, et al. Definition of osteoarthritis of the knee for epidemiological studies. Ann Rheum Dis 1993;52:790-4.
25. Auleley GR, Giraudeau B, Dougados M, et al. Radiographic assessment of hip osteoarthritis progression: impact of reading procedures for longitudinal studies. Ann Rheum Dis 2000;59:422-7.

26. Reijman M, Hazes JM, Koes BW, et al. Validity, reliability, and applicability of seven definitions of hip osteoarthritis used in epidemiological studies: a systematic appraisal. Ann Rheum Dis 2004;63:226-32.

27. Brouwer GM, van Tol AW, Bergink AP, et al. Association between valgus and varus alignment and the development and progression of radiographic osteoarthritis of the knee. Arthritis Rheum 2007;56:1204-11.

28. Zhang $\mathbf{Y}$, Niu J, Felson DT, et al. Methodologic challenges in studying risk factors for progression of knee osteoarthritis. Arthritis Care Res (Hoboken) 2010;62:1527-32.

29. Clockaerts S, Bastiaansen-Jenniskens YM, Runhaar J, et al. The infrapatellar fat pad should be considered as an active osteoarthritic joint tissue: a narrative review. Osteoarthr Cartil 2010;18:876-82.

30. Runhaar J, Koes BW, Bierma-Zeinstra SM. Obesity and biomechanics of every day movements; a systematic review. Osteoarthritis Cartilage 2009;17(Suppl 1):S91.

31. Reijman M, Pols HA, Bergink AP, et al. Body mass index associated with onset and progression of osteoarthritis of the knee but not of the hip: the Rotterdam Study. Ann Rheum Dis 2007;66:158-62.

32. Grotle M, Hagen KB, Natvig B, et al. Obesity and osteoarthritis in knee, hip and/ or hand: an epidemiological study in the general population with 10 years follow-up. BMC Musculoskelet Disord 2008;9:132.

33. Bierma-Zeinstra SM, Koes BW. Risk factors and prognostic factors of hip and knee osteoarthritis. Nat Clin Pract Rheumatol 2007;3:78-85.

34. Beattie MS, Lane NE, Hung YY, et al. Association of statin use and development and progression of hip osteoarthritis in elderly women. J Rheumatol 2005;32:106-10.

35. Chodick G, Amital H, Shalem Y, et al. Persistence with statins and onset of rheumatoid arthritis: a population-based cohort study. PLoS Med 2010; 7:e1000336.

36. Mazzuca SA, Brandt KD, Buckwalter KA. Detection of radiographic joint space narrowing in subjects with knee osteoarthritis: longitudinal comparison of the metatarsophalangeal and semiflexed anteroposterior views. Arthritis Rheum 2003:48:385-90.

37. Abe M, Matsuda M, Kobayashi $\mathrm{H}$, et al. Effects of statins on adipose tissue inflammation: their inhibitory effect on MyD88-independent IRF3/IFN-beta pathway in macrophages. Arterioscler Thromb Vasc Biol 2008;28:871-7.

38. Dollery CM, McEwan JR, Henney AM. Matrix metalloproteinases and cardiovascular disease. Circ Res 1995;77:863-8.

39. Nissen SE. Effect of intensive lipid lowering on progression of coronary atherosclerosis: evidence for an early benefit from the Reversal of Atherosclerosis with Aggressive Lipid Lowering (REVERSAL) trial. Am J Cardiol 2005;96:61F-8F.

40. Palmer G, Chobaz V, Talabot-Ayer D, et al. Assessment of the efficacy of different statins in murine collagen-induced arthritis. Arthritis Rheum 2004:50:4051-9.

41. Schoofs MW, Sturkenboom MC, van der Klift M, et al. HMG-CoA reductase inhibitors and the risk of vertebral fracture. J Bone Miner Res 2004;19:1525-30.

42. Dahaghin S, Bierma-Zeinstra SM, Koes BW, et al. Do metabolic factors add to the effect of overweight on hand osteoarthritis? The Rotterdam Study. Ann Rheum Dis 2007:66:916-20 


\section{Corrections}

S Clockaerts, G J V M Van Osch, Y M Bastiaansen-Jenniskens, et al. Statin use is associated with reduced incidence and progression of knee osteoarthritis in the Rotterdam study. Ann Rheum Dis 2012;71:642-647.

The following text should have been removed from the main body of the article and added underneath the tables by way of an explanation of the tables:

Table 2. Statin use was defined as the use of statins for 120 days or greater, and $50 \%$ or greater of the daily recommended intake. Data are means (SD) or $\mathrm{n}(\%)$. Subjects with missing values were excluded from the analysis. Osteoarthritis is defined as a K\&L score of 2 or more. All subjects with baseline statin use, rheumatoid arthritis, gout, Bechterew disease, femur fracture (for knee osteoarthritis) and hip fracture (for hip osteoarthritis) were excluded.

Table 3. Adjusted OR, 95\% CI and $\mathrm{p}$ values were computed by multivariate analysis with generalised estimating equations to account for left and right correlations. The OR were adjusted for the baseline K\&L score, gender, age, BMI, femoral BMD, total cholesterol/HDL ratio, current smoking, diabetes mellitus, peripheral artery disease, arterial hypertension, educational level and months between baseline and follow-up. Statin use was defined as the use of statins of 120 days or greater, and $50 \%$ or greater of the daily recommended intake. To analyse the effect of increasing cumulative exposure, three intervals of statin use were defined for subjects with an average daily intake of $50 \%$ or greater: 1-119, 120-364 and 365 days or greater, whereas all other subjects were defined as non-users.

We apologise for this error and for any inconvenience this may have caused.

Ann Rheum Dis 2012;71:1264. doi:10.1136/annrheumdis-2011-200092corr1 